\title{
Long-standing Blades of Scissors in paranasal sinus over 40 years
}

\author{
Ji-Eun Choi ${ }^{1}$, Hack Jung Kim² ${ }^{2}$, and Seung-Kyu Chung ${ }^{3}$ \\ ${ }^{1}$ Affiliation not available \\ ${ }^{2}$ Samsung Medical Center \\ ${ }^{3}$ Samsung Medical Center, Sungkyunkwan University School of Medicine
}

April 28, 2020

\begin{abstract}
Herein, we report an unusual case of metallic foreign body consisting of blades of scissors in the maxillary sinus for about 40 years. Even though there are potential risk for sinus infection, not all retained foreign body require prompt removal.
\end{abstract}

\section{Long-standing Blades of Scissors in paranasal sinus over 40 years}

Running title: Blades of scissors lodged in maxillary sinus

Authors: Ji-Eun Choi, Hack Jung Kim, Seung-Kyu Chung

Affiliation:

Department of Otorhinolaryngology-Head and Neck Surgery, Samsung Medical Center, Sungkyunkwan University School of Medicine, Seoul, Republic of Korea

Corresponding author: Seung-Kyu Chung MD. PhD.

Department of Otorhinolaryngology-Head and Neck Surgery, Samsung Medical Center, Sungkyunkwan University School of Medicine,

81 Irwon-ro, Gangnam-gu, Seoul, 06352, Republic of Korea

TEL: 82-2-3410-3579/ FAX: 82-2-3410-6987

E-mail: rhinochung@gmail.com

\section{Key Clinical Message}

Paranasal foreign bodies are infrequent clinical entities. The presence of foreign body often require surgical removal but there are no consensus on clinical indications. This case illustrates the surgical implications of foreign bodies in sinus.

\section{Keywords}

Foreign body; Sinus; Maxillary sinus; Endoscopy

\section{INTRODUCTION}

There are a limited number of reports in the literature that document foreign bodies in the paranasal sinus. Additionally, there are limited reports on the natural clinical course and impact of foreign bodies in the maxillary sinus. ${ }^{1}$ Such clinical situations represent a unique challenge for surgeons. Treatment guidelines about this uncommon phenomenon are not well established because prospective or controlled studies are not feasible due to the limited number of subjects. Guidelines on some factors have been reported by surgeons in 
regard to the location of the foreign body, proximity to vital neurovascular anatomical structures, duration of foreign body impact, and risk of surgical retrieval. ${ }^{1,2}$

In this article, we describe an unusual case of a transfacial penetrating injury with a metallic foreign body (blades of scissors) that remained asymptomatic for about 40 years and was successfully removed via an endoscopic approach. Furthermore, we aim to address issues about management of foreign bodies in the sinonasal cavity to provide guidance and aid in decision-making and treatment processes.

\section{CASE PRESENTATION}

A 61-year-old man presented at the clinic with a one-year history of foul-smelling nasal drainage and progressive nasal congestion. The physical examination was unremarkable except purulent discharge from the left middle meatus based on nasal endoscopy. Standard radiographs showed a metallic shadow in the left maxillary sinus, as presented inFigure 1 . Computed tomography $(\mathrm{CT})$ scans further demonstrated presence of metallic foreign bodies that looked similar to blades of scissors inside the left maxillary sinus and that were obstructing the middle meatus (Figure 2 A (front) \& B (lateral)). History revealed a facial stabbing accident by a senior military officer about 40 years prior. There was a non-notable healed scar at the area of entry.

After precise assessment, the location and direction of the foreign body were determined, and we planned to remove the object with an endoscopic approach; removal via the Cald-well Luc approach was planned as a secondary option. The patient underwent functional endoscopic sinus surgery under general anesthesia, and the stained metallic foreign bodies were successfully removed intranasally (Figure 3 ). The metallic foreign bodies were determined to be rusty blades of scissors (Figure 3 ). There were no postoperative complications during or after the surgery, and the patient noted immediate symptomatic improvement. There were no infectious complications during the follow-up period. The nasal cavity had completely healed after 3 months, and the patient remained asymptomatic without complications.

\section{DISCUSSION}

A foreign body in the paranasal sinus is an uncommon clinical presentation. The condition is primarily associated with previous history of surgery, maxillofacial trauma, or dental procedures. The most common site of involvement is the maxillary sinus. Ethmoidal and sphenoid sinus foreign bodies are extremely rare. After diagnosis, the primary treatment modality is surgical retrieval, and the approach is typically along the course of penetration. Prior reviews have suggested treatment algorithms according to clinical scenario. Since foreign bodies in both symptomatic and asymptomatic cases may serve as a permanent source of irritation, it seemed reasonable to remove the object to prevent irritation and inflammation of the paranasal sinus. However, this case that was asymptomatic for 40 years emphasizes the importance of questioning and considering the true indications of surgical removal of foreign bodies in the paranasal sinus.

There are only a few cases that have reported foreign body impaction for more than 20 years. ${ }^{3,4}$ Lee et al. ${ }^{4}$ reported maxillary sinus fungus ball development caused by retained foreign bodies and that was asymptomatic for 25 years and was successfully removed via a combined endoscopic and Caldwell-Luc approach. In addition, Kuhnel et al. ${ }^{3}$ reported an air gun pellet that had remained in the maxillary sinus for 50 years. Retained foreign bodies theoretically result in infectious complications, such as sinusitis. ${ }^{4,5}$ However, the actual clinical course of foreign body lodgment remains unclear. Prior reports of paranasal foreign bodies have identified pencils, paintbrushes, cues, chopsticks, ball pens, wire, and plastic sticks. Most cases consist of a metallic or plastic foreign body. A metallic foreign body comprised of a toxic element, such as a leadcontaining bullet, prompts urgent retrieval due to the possibility of blood poisoning. To date, no reports have discussed the surgical necessity to treat other kinds of substances. Natural substances such as wood and plants are highly associated with infection, so prompt removal is indicated. In comparison, plastic foreign bodies do not tend to induce inflammation. There is currently no consensus for metallic foreign bodies other than those containing lead. We were not able to analyze the exact composites of the scissor blades but hypothesize that the materials differ from those, such as stainless steel, currently used to make scissors. Stainless steel consists of nickel or iron, and the advances in technology could have impacted the corrosivity. 
Therefore, it is possible that a foreign body consisting of stainless steel might not directly cause critical inflammation, and immediate surgery may not be necessary. For example, cases that could wait and be kept under observation would include instances where the surgical removal approach is particularly difficult, if the foreign body is not in close proximity to neurovascular structures, or if the patient refuses surgery. Additionally, multiple fragments of foreign bodies may not be implicated for surgical removal. Therefore, surgeons must weigh benefits and harms and carefully discuss with the patient. If surgeons opt for an observation approach, there is a secondary issue about the relevant artifacts that may hinder precise evaluation when performing computed tomography or MRI. This must be also discussed with the patient to optimize treatment and outcomes.

\section{CONCLUSIONS}

In this paper, we reported a case of an unusual metallic foreign body identified in the sinus cavity and that remained asymptomatic for about 40 years. Although there are potential risks for sinus infection, not all retained foreign bodies require immediate removal. Therefore, timing and approach for retrieval should be considered and validated by additional studies. In general, foreign bodies that can easily be removed by an endoscopic approach may prompt surgical removal, while cases with significant risks associated with removal may warrant an observation approach.

\section{CONFLICT OF INTEREST}

None declared

\section{AUTHOR CONTRIBUTIONS}

All authors have made substantial contributions to all of the following.

JEC : collected clinical data and conceived the draft of the manuscript

HJK : collected clinical data and participated in the management of this patient

SKC: participated in the management of this patient as well as in the preparation and edition of this manuscript, read and approved the final manuscript.

\section{REFERNCES}

1. Yarlagadda B, Jalisi S, Burke P, Platt M. Retrieval of projectile foreign bodies from the paranasal sinuses and skull base. Am J Rhinol Allergy 2012;26:233-236.

2. Dominguete PR, Matos BF, Meyer TN, Oliveira LR. Jael syndrome: removal of a knife blade impacted in the maxillofacial region under local anaesthesia. BMJ Case Reports 2013;2013:bcr-2013-008839.

3. Kuhnel TV, Tudor C, Neukam FW, Nkenke E, Stockmann P. Air gun pellet remaining in the maxillary sinus for 50 years: a relevant risk factor for the patient? Int J Oral Maxillofac Surg 2010;39:407-411.

4. Lee DH, Lim SC. Maxillary fungus ball caused by retained foreign bodies for 25 years. J Craniofac Surg 2012;23:e176-177.

5. Dimitrakopoulos I, Papadaki M. Foreign body in the maxillary sinus: report of an unusual case. Quintessence Int 2008;39:698-701.

\section{FIGURE LEGENDS}

Figure 1. Preoperative anteroposterior (A) and lateral(B) x-rays showing a radiopaque maxillary sinus foreign body.

Figure 2. Preoperative CT scan demonstrating the location of metallic foreign body (blades of scissors) (coronal;A, axial;B)

Figure 3. (A) Endoscopic image of the foreign body retained in middle meatus during surgery. 
Two sets of blades were successfully removed via endoscopic approach.

(asterisk showing septum, arrow designating middle turbinate)

(B) Photograph demonstrate the rusted metallic foreign body removed by surgery.
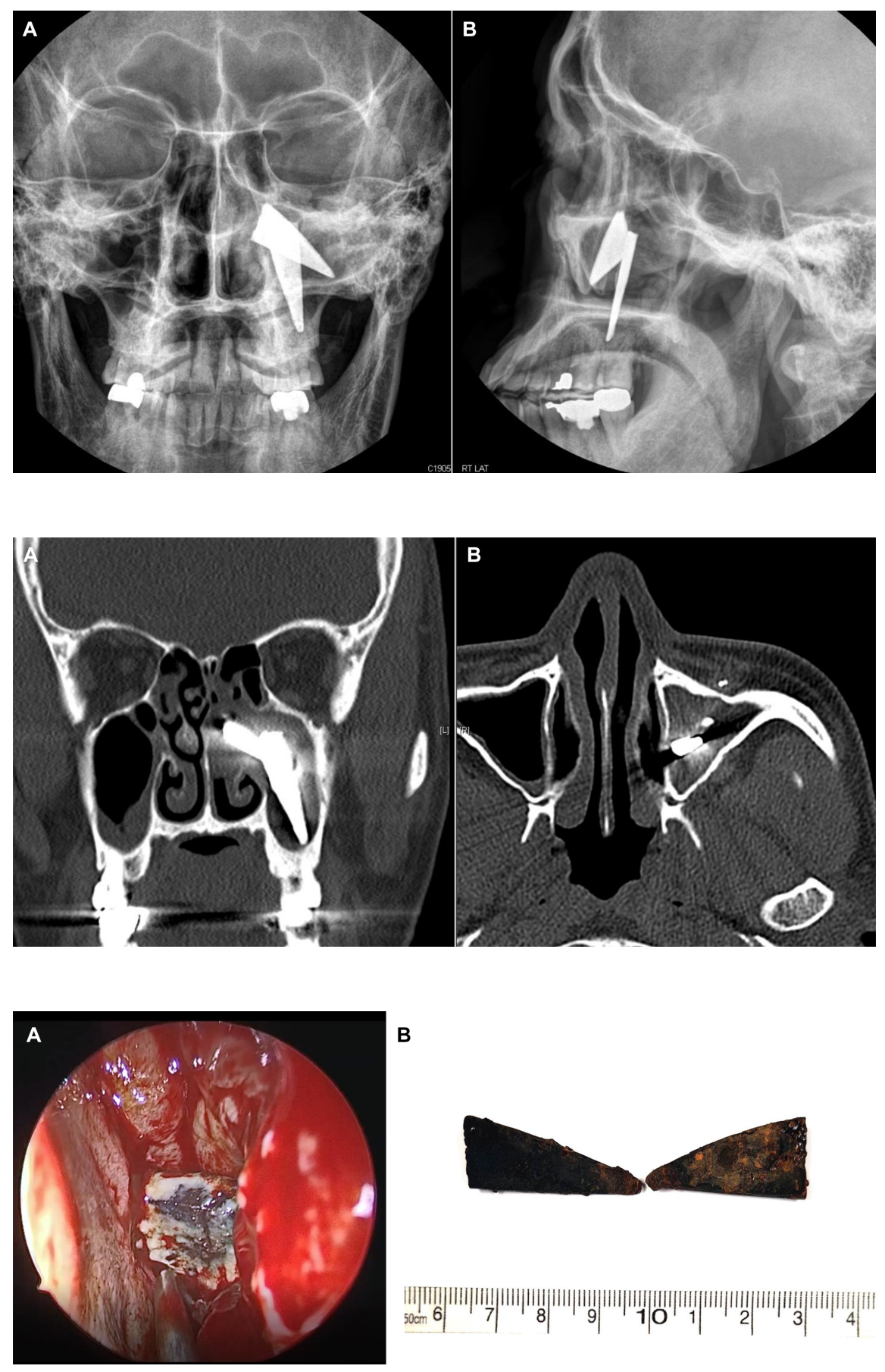

B

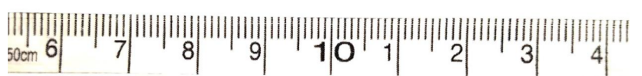

